\title{
In memoriam Prof. Dr. med. Karl Wurm (1906 - 2005) u. costabel
}

Prof. Dr. med. Karl Wurm (1906-2005) in Memoriam

Am 10. Juli 2005 verstarb Prof. Dr. Karl Wurm im biblischen Alter von 98 Jahren. Als deutscher Sarkoidosepionier erwarb er sich internationale Anerkennung. Seine mehr als ein halbes Jahrhundert andauernde Auseinandersetzung mit der Sarkoidose begann 1950 in Höchenschwand im Schwarzwald, dem „Dorf am Himmel“. Hier erkannte er als Internist und frisch berufener Chefarzt der Augenheilstätte frühzeitig, dass die Augenerkrankungen seiner Patienten häufig auf die Sarkoidose zurückzuführen waren. Sein bleibendes Verdienst besteht in der Einteilung der Sarkoidose in Röntgenstadien, welche noch heute ihre Gültigkeit haben und in ihrer prognostischen Aussage von keinem modernen Biomarker übertroffen werden. Bis ins hohe Alter stellte er seine reichen Erfahrungen vielen Sarkoidosepatienten zur Verfügung.

Karl Wurm wurde am 13. November 1906 als sechstes von sieben Kindern in Bergheim bei Dillingen an der Donau geboren, einem

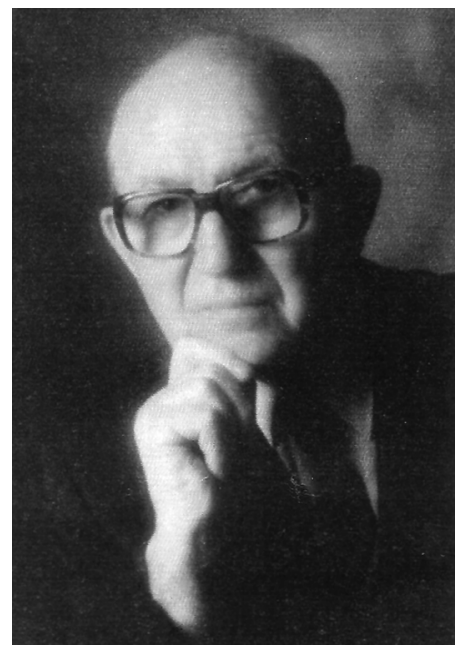

Prof. Dr. med. Karl Wurm einfachen Bauerndorf des damaligen Bayerischen Königreiches. Sein Vater war Landwirt und Sattler. Nach einigen Schuljahren auf dem bischöflichen Knabenseminar in Dillingen wurde ihm bewusst, dass das ursprüngliche Berufsziel Pfarrer für ihn nicht das Richtige war. Nach einer Tätigkeit am Finanzamt in Augsburg wurde er zum Medizinstudium durch die Buchlektüre P. H. Kruif „Der Mikrobenjäger" angeregt und verdiente sich sein Studium in Freiburg und München selbst, unter anderem auch als Werkstudent in den Kalibergwerken von Buggingen bei Freiburg. Bereits nach 9 Studiensemestern absolvierte er 1932 das medizinische Staatsexamen und promovierte gleichzeitig mit dem Prädikat „magna cum laude“. Nach einer dreijährigen Assistenzzeit bei Geheimrat Prof. Uhlenhut am Freiburger Hygieneinstitut habilitierte er sich 1937 mit einer Arbeit über Lymphogranulomatose, in der es ihm gelang, die Hypothese einer infektiösen
Ätiologie des Morbus Hodgkin zu widerlegen. Er war damals der jüngste habilitierte Arzt der Freiburger Universitätsklinik.

Als junger Wissenschaftler wechselte er von Freiburg nach Prag, wo er 1940 eine Dozentur für Innere Medizin erhielt. Während des Krieges wurde er als beratender Sanitätsoffizier für die Seuchenbekämpfung eingesetzt und kam mit dem Zusammenbruch des Deutschen Reiches in russische Kriegsgefangenschaft, aus der er erst 1949 zurückkehrte. Die Tätigkeit als Chefarzt der privaten Kuranstalten Augenheilstätte Sonnenhof und Kurhaus Höchenschwand wurde von ihm und seiner charmanten Ehefrau Hanna, einer gebürtigen Wienerin, zunächst nur als vorübergehende Interimslösung angesehen. Die Beschäftigung mit den Au- 
genpatienten führte jedoch dazu, dass er das Krankengut der Sarkoidose entdeckte und sein Leben lang davon gefesselt blieb. Bis dahin war der "Morbus Boeck“ weder ihm selbst noch der allgemeinen Ärzteschaft bekannt. Höchenschwand wurde ihm so zur zweiten Heimat. In engem Kontakt zur Freiburger Universität (Professores Reindell, Doll, Kalkoff, Lesch, Matthys) und zu anderen Instituten (Universität Zürich, Prof. Uehlinger) wurde ihm der wissenschaftliche Umgang mit der Sarkoidose zum Lebensinhalt. Er hielt regelmäßige Vorlesungen an der Medizinischen Universitätsklinik Freiburg, wo er 1954 zum Professor für Innere Medizin ernannt wurde. 1954 erschien auch die erste Sarkoidosearbeit, gemeinsam mit $\mathrm{H}$. Reindell über klinisch/röntgenologische Untersuchungen bei Morbus Boeck, publiziert in der klinischen Wochenschrift, und 1958 die bahnbrechende Monographie „Der Lungenboeck im Röntgenbild“, mit H. Reindell und L. Heilmeyer als Koautoren. Sein ärztliches Interesse am Patienten und seine publizistische Aktivitäten erlahmten bis ins hohe Alter nicht. Bis 1997 waren es weit mehr als 100 Publikationen über die Sarkoidose, die aus seiner Feder stammten.

Niemand in Deutschland übersah ein so großes Krankengut an Sarkoidose und befasste sich so intensiv mit der Erforschung des Verlaufs und der Behandlung dieses Krankheitsbildes wie Karl Wurm. Zehntausende von Sarkoidosepatienten kamen zu ihm nach Höchenschwand in das von ihm gegründete Sarkoidosezentrum. Zahlreiche Gäste aus dem In- und Ausland holten sich hier Anregungen.

Bereits früh pflegte Karl Wurm internationale Kontakte. So nahm er als einer von 22 Ärzten am ersten internationalen Sarkoidosetreffen 1958 in London teil, aus dem später die regelmäßigen internationalen Sarkoidosekonferenzen hervorgingen. Nationale und internationale Auszeichnungen blieben nicht aus. Ihm wurden Ehrungen wie das Bundesverdienstkreuz am Bande, das Fakultätswappen der Medizinischen Fakultät Tokyo, die Ehrenmitgliedschaften der Selbsthilfegruppe „Deutsche Sarkoidosevereinigung“, der Japanischen Sarkoidosegesellschaft und der „World Association of Sarcoidosis and Other Granulomatous Disorders" sowie der Presidential Award dieser Gesellschaft anlässlich des Sarkoidose-Weltkongresses in Essen verliehen. 1996 ehrte ihn die Gemeinde Höchenschwand durch das Namenspatronat „Professor-Wurm-Straße“ (Ärztlicher „Vater“ des Kurortes nach dem 2. Weltkrieg).

Karl Wurm wird uns als Nestor der Deutschen Sarkoidoseforschung, als bescheidener Mensch und großartiger Arzt, der immer von der Sorge um seine Patienten getragen war, unvergessen bleiben. 